\title{
锂离子电池陶瓷复合全固态电解质的制备和性能研究
}

\author{
李承斌 ${ }^{1,2}$, 岳红云 ${ }^{3}$, 王秋娴 ${ }^{3}$, 李静娴 ${ }^{3}$, 杨书廷 ${ }^{1,3}$
}

(1. 河南师范大学 物理与材料科学学院, 新乡 $453007 ; 2$. 河南工学院 电气工程系, 新乡 $453003 ; 3$. 河南师范大学 化学化工学院, 动力电源及关键材料国家地方联合工程实验室, 新乡 453007)

摘 要: 以负载 $\mathrm{Al}_{2} \mathrm{O}_{3}$ 的无纺布为支撑膜, 浸涂 PEO-LAGP-SN-LiTFSI 的乙腈共混液干燥后制得新型复合固态电解 质膜(CLASP)。该膜的热稳定性好, 即使在 $170^{\circ} \mathrm{C}$ 的高温下依然不发生形变。当浸涂共混液中 PEO: LAGP: SN: LiTFSI 为 3: 1: $1: 1$, 固含量为 $10 \mathrm{wt} \%$ 时, 室温电导率可以达到 $3.66 \times 10^{-5} \mathrm{~S} / \mathrm{cm}, 100^{\circ} \mathrm{C}$ 时电导率可达 $2.52 \times 10^{-4} \mathrm{~S} / \mathrm{cm}$. CLASP 膜的电化学窗口宽, 以该膜代替液态电解质装配的全固态 $\mathrm{LiFePO}_{4} / \mathrm{CLASP} / \mathrm{Li}$ 电池, 在 $55^{\circ} \mathrm{C}$ 循环时表现出良 好的循环稳定性, 高的库伦效率, 有望成为电化学性能优越的全固态电解质。

关 键 词: 全固态; 复合电解质; 磷酸锗铝锂; 耐热

中图分类号: Q152 文献标识码: A

\section{Preparation and Property of a Novel Heat-resistant Ceramic Composite Solid-state Electrolyte for Lithium Batteries}

\author{
LI Cheng-Bin ${ }^{1,2}$, YUE Hong-Yun ${ }^{3}$, WANG Qiu-Xian ${ }^{3}$, LI Jing-Xian ${ }^{3}$, YANG Shu-Ting ${ }^{1,3}$ \\ (1. College of Physics and Materials Science, Henan Normal University, Xinxiang 453007, China; 2. Department of Electrical \\ Engineering, Henan Institute of Technology, Xinxiang 453003, China; 3. National and Local Joint Engineering Laboratory of \\ Motive Power and Key Materials, School of Chemistry and Chemical Engineering, Henan Normal University Xinxiang 453007 , \\ China)
}

\begin{abstract}
Poly(ethylene oxide) (PEO) as a kind of promising candidates of polymer electrolyte is widely applied in all solid lithium ion batteries. In this study, a novel ceramic composite solid electrolyte (CLASP) was prepared by dip-coating PEO-LAGP-SN-LiTFSI (PEO-SPE) into $\mathrm{Al}_{2} \mathrm{O}_{3}$ nonwoven membrane. And all solid batteries were assembled with CLASP. CLASP showed excellent thermostability even at $170^{\circ} \mathrm{C}$ for $2 \mathrm{~h}$. When the ratio of PEO: LAGP: SN: LiTFSI was 3: $1: 1: 1$, the conductivities of CLASP reached $3.66 \times 10^{-5} \mathrm{~S} / \mathrm{cm}$ at $25^{\circ} \mathrm{C}$ and $2.52 \times 10^{-4} \mathrm{~S} / \mathrm{cm}$ at $100^{\circ} \mathrm{C}$, respectively. The electrochemical window of CLASP was $5.5 \mathrm{~V}$, which had great potential to apply in battery with high voltage cathode material. The cycling performance of all solid batteries with CLASP was greatly improved at $55^{\circ} \mathrm{C}$ as well. This new ceramic composite, solid polymer electrolyte, with all these outstanding performance is a promising candidate of all solid electrolytes.
\end{abstract}

Key words: all solid state; composite electrolyte; LAGP; heat-resistance

自从 1991 年索尼开发出商用锂离子电池以来, 锂离子电池以其具有高的能量密度，无记忆效应，
环境友好等特点被广泛应用于手机和笔记本等数码 产品中 ${ }^{[1-3]}$ 。但是它一直存在安全问题 ${ }^{[4-6]}$, 造成锂离

收稿日期：2016-11-07; 收到修改稿日期：2017-02-17

基金项目：国家自然科学基金(U1504211); 河南省科技攻关项目(142102210449)

National Natural Science Foundation of China (U1504211); Science and Technology Project of Henan Province (142102210449)

作者简介: 李承斌(1984-), 男, 博士研究生. E-mail: milestone31@foxmail.com

通讯作者：岳红云，副教授.E-mail: yuehongyun@foxmail.com; 杨书廷，教授. E-mail: shutingyang@foxmail.com 
子电池安全问题主要是由于商业化锂电中含有大量 的有机液态电解质, 在充电过程中电池温度急剧升 高, 液态电解质在高温下受热分解产生气体, 易使 电池电解液泄漏, 同时在高温下聚烯烃隔膜会有一 定收缩, 最终使电池发生短路或爆炸 ${ }^{[7-9]}$ 。因此, 固态 电解质可以从根本上解决锂离子电池安全问题, 而且 应用固态电解质的全固态锂离子电池可以直接用金 属锂做负极, 能极大的提高电池的能量存储 ${ }^{[10-12]}$ 。因 此对于固态电解质的研究备受人们的关注。

目前研究较为广泛的固态电解质包括无机固态 电解质和聚合物固态电解质, 其中常用的无机固体 电解质有钻钛矿类、锂超离子导体类(LISICON)、钠 超离子导体类 $(\mathrm{NASICON}) 、$ 层状 $\mathrm{Li}_{3} \mathrm{~N}$ 类、 $\mathrm{LiPON}$ 类 以及硫化物等 ${ }^{[13-14]}$ 。本实验室制备了具有 NASICON 结构的磷酸锗铝锂, 即 $\mathrm{Li}_{1.5} \mathrm{Al}_{0.5} \mathrm{Ge}_{1.5}\left(\mathrm{PO}_{4}\right)_{3}$ ( $\left.\mathrm{LAGP}\right)$, 它属于快离子导体, 具有较好的电化学性能, 不仅 耐高温, 而且在空气中也非常稳定, 但是如果直接 用 LAGP 粉体材料压片用做锂电的固态电解质, 由 于它与电极材料间属于固-固接触, 电性能不佳 ${ }^{[15]}$; 而加入可传导锂离子的聚合物可以克服固-固界面 接触性差的不足, 因此本工作将 LAGP 加入到聚合 物聚氧化乙烯(PEO)、导电锂盐双三氟甲烷磺酰亚胺 锂(LiTFSI)和塑化剂丁二腈(SN)体系中, 共同组成 固体电解质体系(PEO-SPE)。为了增强固体电解质 的机械性能和阻然性, 采用孔隙较大的双面负载 $\mathrm{Al}_{2} \mathrm{O}_{3}$ 的无纺布 (FS-25) 作为支撑膜, 它不仅能提供 力学支撑, 而且可以填充复合电解质形成连续相, 起到传导锂离子的作用。在无纺布两面均匀浸涂 PEO-LAGP-SN-LiTFSI 的乙腈共混液, 干燥后制得 固态复合电解质膜(CLASP), 并用 CLASP 组装全固 态锂离子电池, 对它们的性能进行测试。

\section{1 实验方法}

\section{1 样品制备}

LAGP 的制备: 将 $\mathrm{Al}\left(\mathrm{NO}_{3}\right)_{3} .9 \mathrm{H}_{2} \mathrm{O} 、 \mathrm{GeO}_{2}$ 、 $\mathrm{NH}_{4} \mathrm{H}_{2} \mathrm{PO}_{4}$ 用 $\mathrm{Li}_{1.5} \mathrm{Al}_{0.5} \mathrm{Ge}_{1.5}\left(\mathrm{PO}_{4}\right)_{3}$ 的化学计量比 0.5: 1.5: 3 称取, $\mathrm{LiOH} \cdot \mathrm{H}_{2} \mathrm{O}$ 过量称取 $5 \% \sim 10 \%$, 原料 球磨混匀后置于 $400^{\circ} \mathrm{C}$ 炉中保温 $2 \mathrm{~h}, 900^{\circ} \mathrm{C}$ 炉中保温 $12 \mathrm{~h}$, 退火, 可得 LAGP。将产物球磨 $24 \mathrm{~h}$ 备用。 LAGP 粉末在 $20 \mathrm{MPa}$ 下压片, $900^{\circ} \mathrm{C}$ 烧结喷金备用。

正极片的制备: 使用 $\mathrm{PEO}$ (分子量 600000)做为 粘结剂。按 $\mathrm{LiFePO}_{4}$ : Super P: PEO = 8: 1: 1 称取各 种原料, 加入乙腈球磨 $2 \mathrm{~h}$ 后把浆料涂于铝簿上, 真 空干燥 $12 \mathrm{~h}$ 后制得正极片。
复合固态电解质膜的制备，按 $\mathrm{PEO}$ (分子量 600000): LiTFSI: SN: LAGP=3: 1: 1: $1 、 \mathrm{EO}: \mathrm{Li}^{+}=19: 1$, 称取原料, 加入乙腈摚拌均匀, 使得总的固含量为 $10 \mathrm{wt} \%$ 。采用双面浸涂法，将配制的固体电解质溶液 均匀浸涂在负载 $\mathrm{Al}_{2} \mathrm{O}_{3}$ 的无纺布 FS-25 的两面, 为了 避免真空干燥时产生气泡和孔隙, 先在室温下静置 $20 \mathrm{~min}$, 待溶剂挥发后, 再在 $60^{\circ} \mathrm{C}$ 真空干燥 $12 \mathrm{~h}$ 制得 固态复合电解质膜 CLASP。将制备的正极、CLASP 电解质膜和金属锂片组装成全固态电池。

\section{2 表征}

使用 JSM-6700F 场发射扫描电镜观察样品的形 貌，所有样品均进行喷金处理，截面用 GATAN Ilion II 697 氩离子束仪切割。用 Beckman Coulter LS 230 测试 LAGP 样品的粒度分布。使用 Themo Nicolet 670FT-IR 测量样品的红外光谱, 用 TA Instruments $\mathrm{Q} 2000$ 对样品从 $-75^{\circ} \mathrm{C} \sim 150^{\circ} \mathrm{C}$ 进行 DSC 热分析, 使用 Bruker AXS D8 测试样品的 XRD 图谱。用 CHI760B 电化学工作站测试固态电解质的交流阻抗谱, 进行线 性伏安扫描, 并计算电导率。使用 CT2001A 在 $55^{\circ} \mathrm{C}$ 下对全固态电池进行电化学充放电测试。

\section{2 结果与讨论}

\section{1 结构与形貌}

图 1(a)是不同样品的红外光谱图, LAGP 在 1107 和 $1041 \mathrm{~cm}^{-1}$ 只有 $\mathrm{P}-\mathrm{O}$ 的伸缩振动吸收峰, 而 FS-25 膜表面含有 $\mathrm{Al}_{2} \mathrm{O}_{3}$, 所以没有明显的吸收峰。PEO 的 红外图谱中有 3 个 $\mathrm{C}-\mathrm{O}-\mathrm{C}$ 的伸缩振动峰分布于

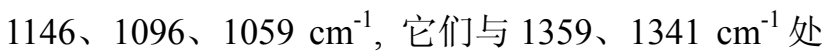
的双峰都来自于 $\mathrm{PEO}$ 结晶相 ${ }^{[16]}$, 而随着塑化剂 $\mathrm{SN}$ 和 锂盐 LiTFSI 以及 LAGP 的加入, $1100 \mathrm{~cm}^{-1}$ 处的三重峰 和 $1350 \mathrm{~cm}^{-1}$ 处的双峰都发生了合并(PEO-SPE 所示), 这说明加入 $\mathrm{SN}$ 、锂盐和 LAGP 显著降低了 PEO 的结 晶度, 有利于电解质的离子传输。而复合电解质膜 (CLASP) 在 $1058 \mathrm{~cm}^{-1}$ 处却又分裂出一个肩峰, 说明锂 离子和膜表面的 $\mathrm{Al}_{2} \mathrm{O}_{3}$ 可能有一定的配位作用, 这有 利于锂盐中 $\mathrm{Li}^{+}$脱嵌, 说明 $\mathrm{Al}_{2} \mathrm{O}_{3}$ 可能对于锂离子的传 输也有一定的作用。在 PEO-SPE 的谱线中, $2900 \mathrm{~cm}^{-1}$ 附近出现了 2880、2988 和 $2951 \mathrm{~cm}^{-1}$ 三个峰, 它们是 由 $\mathrm{PEO}$ 和 $\mathrm{SN}$ 中的 $\mathrm{CH}_{2}$ 伸缩振动峰叠加出来的; 而在 CLASP 中这三个峰会在 $2884 \mathrm{~cm}^{-1}$ 处合并, 进一步确 定 PEO 结晶程度的降低, 无定形态的增加。

图 1(b)是 LAGP 粉末的 XRD 图谱, LAGP 属于 六方结构, 它由导电的主晶相 $\mathrm{LiGe}_{2}\left(\mathrm{PO}_{4}\right)_{3}$ 和次晶相 $\mathrm{AlPO}_{4}\left(26^{\circ}\right.$ 处小峰)组成 ${ }^{[17]}$ 。图 1(b)插图为 LAGP 粉 
末的粒径分布图，从图中可以看出, LAGP 颗粒的粒 径主要分布在 120 200 nm 之间。图 1(c)显示的是负 载 $\mathrm{Al}_{2} \mathrm{O}_{3}$ 的无纺布膜浸涂电解质前后的 XRD 图谱, 从图中可以看出浸涂后衍射峰位向高角度偏移, 说 明晶胞收缩, 晶格常数变小, 可能是由于掺入了比 $\mathrm{Al}_{2} \mathrm{O}_{3}$ 颗粒更小的 LAGP 造成的。对比还可发现, 浸 涂电解质后衍射峰的强度减小, 说明浸涂后 FS-25 无纺布的表面上均匀覆盖了 PEO 和 LAGP, 在聚合 物 PEO 和更小的 LAGP 颗粒共同作用下, 结晶化程 度有所降低, 表现为峰强变弱。

图 2 是浸涂前后的场发射扫描电镜照片, 图 2(a)、(b)和 (c)是无纺布浸涂前的表面和截面, 图 2(d)、(e)和(f) 是固态复合电解质膜 CLASP 的表面 和截面。从图 2(a)中可以看出表面的 $\mathrm{Al}_{2} \mathrm{O}_{3}$ 颗粒, 但 是没有覆盖完全, 留有大量孔隙; 从放大的图 2(b) 中可以看出 $\mathrm{Al}_{2} \mathrm{O}_{3}$ 颗粒的粒径大约在 $0.5 \sim 1 \mu \mathrm{m}$, 孔
隙为 $0.1 \sim 0.5 \mu \mathrm{m}$ 。而从浸涂后的图 2(d)中可以看到 膜表面已无明显的孔,表明电解质涂覆比较均匀; 从放大的图 2(e)中能看出 $\mathrm{Al}_{2} \mathrm{O}_{3}$ 颗粒上均匀分布有 更加细小纳米级的 LAGP 颗粒, PEO 可以很好地把 锂盐和 LAGP 粘附在无纺布膜中, 这一点也可从 XRD 图谱右移且峰强度降低加以印证。对比浸涂 前后截面形貌不难发现，浸涂前(图 2(c))的截面存 在大量的裂缝, 且分布广泛, 不利于锂离子在电极 间的迁移传输; 而双面浸涂后的膜(图 2(f))比较紧 实致密, 说明电解质已经将膜的孔隙填满形成了 连续相, 这样就有利于锂离子的迁移, 从而提高电 导率。

\section{2 热分析}

图 3 是不同样品的 DSC 曲线, 测试在 $\mathrm{N}_{2}$ 氛围 下进行, 升温速率为 $10^{\circ} \mathrm{C} / \mathrm{min}$, 从图中可以看出 PEO 在 $67.5^{\circ} \mathrm{C}$ 有一个尖锐的吸热峰, 对应它的熔点,
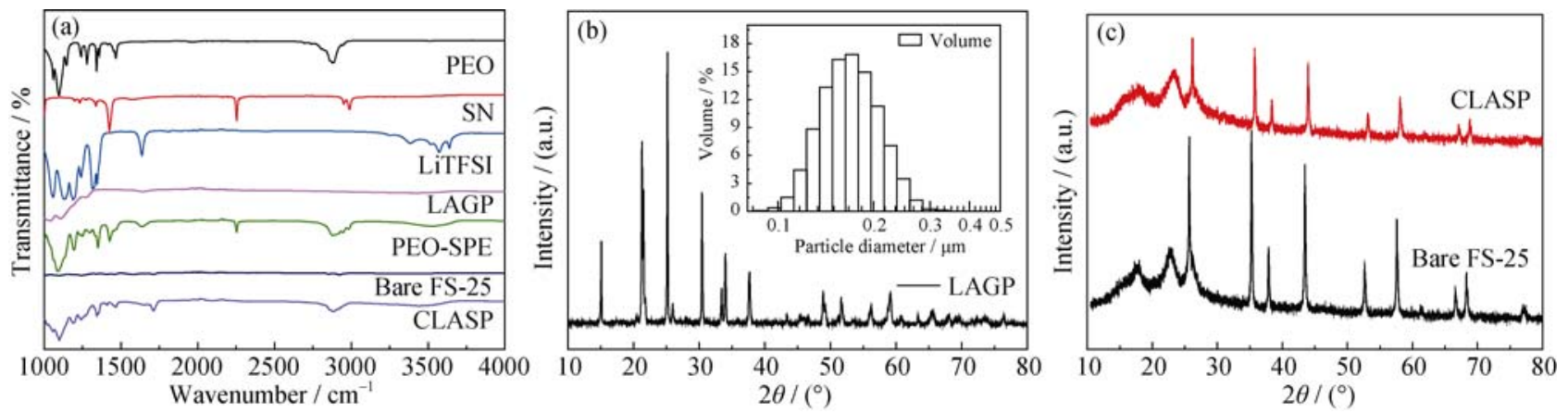

图 1 (a) PEO, SN, LiTFSI, LAGP, PEO-SPE, FS-25 无纺布和 CLASP 的红外光谱图; (b) LAGP 的 XRD 图谱和粒径分布图;

(c) FS-25 无纺布和 CLASP 的 XRD 图谱

Fig. 1 (a) ATR-FTIR spectra of PEO, SN, LiTFSI, LAGP, PEO-SPE, bare FS-25, and CLASP; (b) XRD pattern of LAGP and the LAGP particle diameter distribution (inset); (c) XRD patterns of bare FS-25 and CLASP

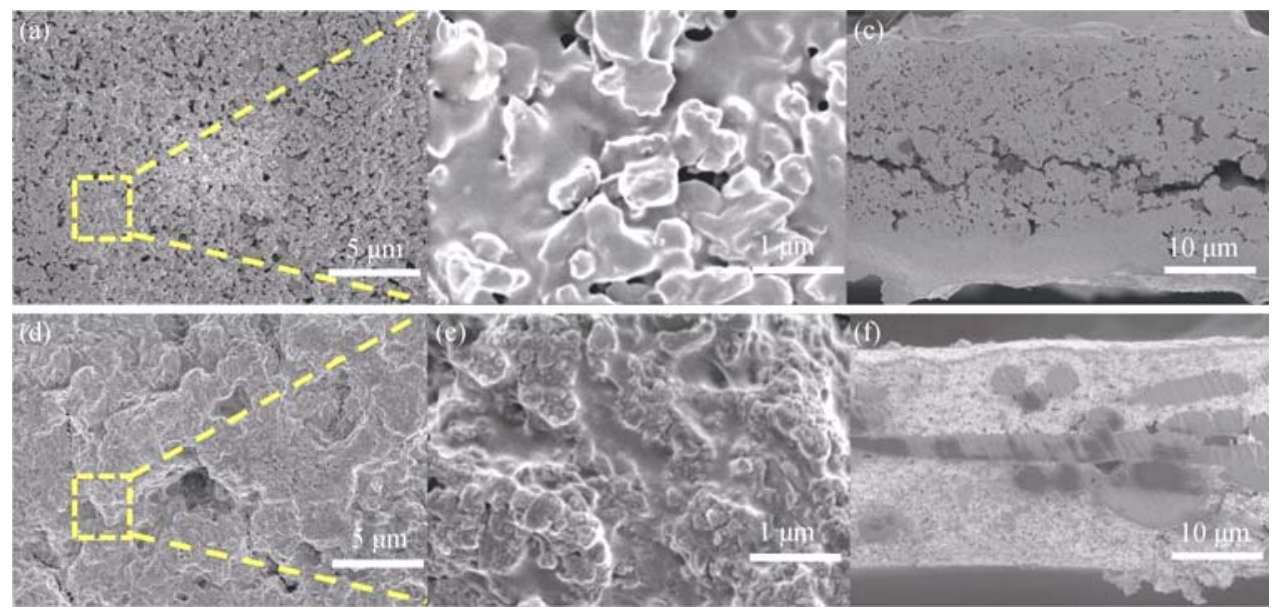

图 2 无纺布 FS-25 和 CLASP 的表面(a,b/d,e)和截面(c/f)的 SEM 照片

Fig. 2 Surface and cross-section SEM images of bare FS-25 (a, b and c) and CLASP (d, e and f) 


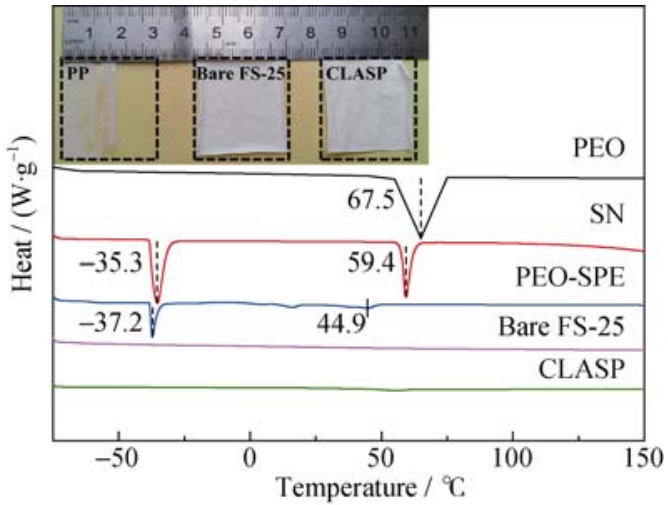

图 3 PEO, SN, PEO-SPE, FS-25 无纺布和 CLASP 的 DSC 曲线

Fig. 3 DSC curves for PEO, SN, PEO-SPE, bare FS-25, and CLASP

Inset is the shrinkage pictures of PP, bare FS-25 and CLASP after treatment at $170^{\circ} \mathrm{C}$ for $2 \mathrm{~h}$

从曲线中可以看出 $\mathrm{PEO}$ 在 $50^{\circ} \mathrm{C}$ 附近就已经开始软 化。塑晶 SN 可以很好地降低聚合物的结晶度, 增加 无定型区的百分比, 从而有利于锂离子的传递，改 善电解质的电性能, 但加入塑晶 SN 后, 电解质 (PEO-SPE)的软化点降到 $44.9^{\circ} \mathrm{C}$, 如果在高温下应用, 电解质就有被锂支晶刺穿发生短路的危险。为了消
除隐患, 采用高温下非常稳定的双面负载 $\mathrm{Al}_{2} \mathrm{O}_{3}$ 的无 纺布做支撑膜(图 3 中 Bare FS-25 没有吸热峰)来提高 电解质的热稳定性和机械性能。从图 3 中可以看出, 浸涂电解质溶液的最终产品 CLASP 没有明显吸热峰, 说明固态电解质膜 CLASP 的耐热性极佳。

从图 3 插图的热收缩实验照片能更直观展现 CLASP 膜的热稳定性，图中从左至右分别是传统电 池用 PP 隔膜和浸涂前后的无纺布膜。实验前把它 们裁成 $3 \mathrm{~cm} \times 3 \mathrm{~cm}$ 的正方形，在 $170^{\circ} \mathrm{C}$ 下加热 $2 \mathrm{~h}$ 后 取出拍照，从照片中可以看出，高温加热后，由于 $\mathrm{Al}_{2} \mathrm{O}_{3}$ 无纺布膜出色的热稳定性, CLASP 膜的面积 无明显变化，而传统的 PP 隔膜面积缩小了一半。说 明复合固态电解质膜的热稳定性良好。

\section{3 电化学分析}

图 4(a)是 LAGP、PEO 基聚合物电解质和 CLASP 膜在 $25 \sim 100^{\circ} \mathrm{C}$ 的离子电导率曲线。从图中可以看出 它们的电导率都随温度升高而增大。其中 LAGP 电 导率最低, 即使在 $100^{\circ} \mathrm{C}$ 也不到 $5 \times 10^{-5} \mathrm{~S} / \mathrm{cm}$ 。而 CLASP 的电导率始终高于 PEO 基聚合物电解质和 LAGP, 而且温度愈高, 差距越大。如在室温 $25^{\circ} \mathrm{C}$ 时, PEO-SPE 和 CLASP 的电导率分别为 $2.35 \times 10^{-5} \mathrm{~S} / \mathrm{cm}$
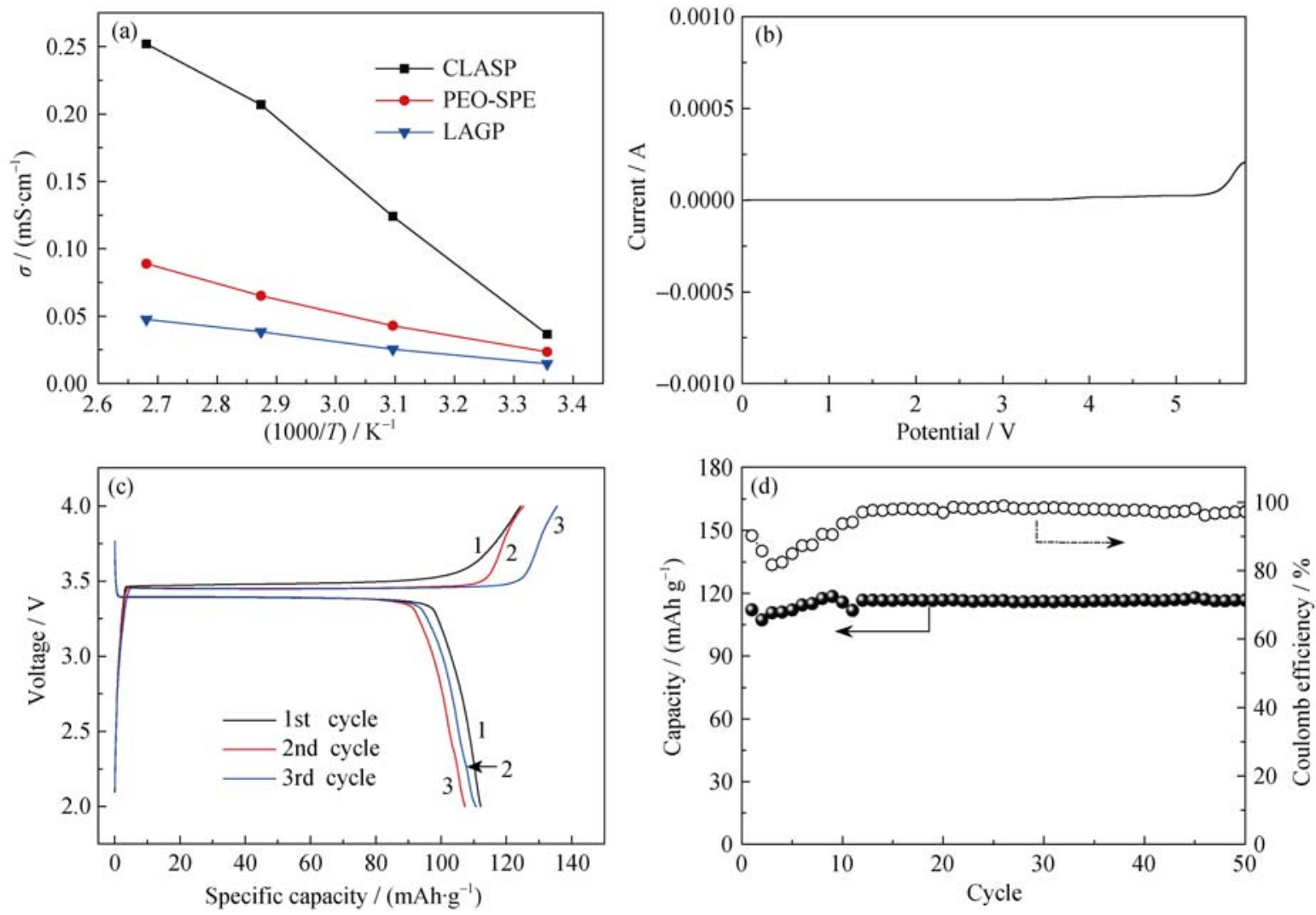

图 4 (a)不同温度下 LAGP、PEO 固态电解质和 CLASP 的离子电导率, (b)CLASP 的线性伏安扫描图, (c) $55^{\circ} \mathrm{C}, 0.1 \mathrm{C}$ 下用 CLASP 组装的全固态电池前三个循环的电压-比容量曲线和 $(\mathrm{d}) 55^{\circ} \mathrm{C}, 0.1 \mathrm{C}$ 下用 CLASP 组装的全固态电池循环图

Fig. 4 (a) Temperature dependence of ionic conductivity for LAGP, PEO-SPE and CLASP; (b) LSV curve for CLASP; the first three charge/discharge voltage profiles $(c)$ and cycling performance $(\mathrm{d})$ of the $\mathrm{LiFePO}_{4} / \mathrm{Li}$ cell using CLASP at $0.1 C, 55^{\circ} \mathrm{C}$ 
和 $3.66 \times 10^{-5} \mathrm{~S} / \mathrm{cm}$; 在 $100^{\circ} \mathrm{C}$ 时, CLASP 的电导率比室 温提升了一个数量级达到 $2.52 \times 10^{-4} \mathrm{~S} / \mathrm{cm}$, 而 PEO$\mathrm{SPE}$ 仅为 $8.89 \times 10^{-5} \mathrm{~S} / \mathrm{cm}$ 。PEO 在室温下具有较高结 晶度, 影响了锂离子的传输, 所以在聚合物中加入 塑晶 SN 和纳米陶瓷颗粒 LAGP 可以在一定程度上降 低结晶度。另外由于无纺布膜负载 $\mathrm{Al}_{2} \mathrm{O}_{3}$ 颗粒的缘故, 可以进一步降低聚合物电解质的结晶程度, 增加无 定型区域的百分比 ${ }^{[18]}$ 。由此可见, 在 SN、LAGP 和 负载 $\mathrm{Al}_{2} \mathrm{O}_{3}$ 的无纺布的共同作用下可以有效提高离 子电导率。

图 4(b)是用不锈钢片和锂片夹住 CLASP 膜组 装成扣式电池, 并进行线性伏安扫描得到的图谱。 从图 4 可以看到, 从 $0 \sim 5.5 \mathrm{~V}$ 没有明显的起伏, 说明 该固态电解质膜的电化学窗口非常宽, 有应用于高 压正极材料的潜力。图 $4(\mathrm{c})$ 是 $55^{\circ} \mathrm{C} 、 0.1 \mathrm{C}$ 条件下全 固态电池 $\mathrm{LiFePO}_{4} / \mathrm{CLASP} / \mathrm{Li}$ 前三次循环的电压一比 容量曲线, 从图中可以看出首次循环比容量为 $112.1 \mathrm{mAh} / \mathrm{g}$, 第二次循环容量略有下降, 第三次循 环容量又有所回升, 放电电压平台在 $3.4 \mathrm{~V}$ 重合性 很好, 符合正极 $\mathrm{LiFePO}_{4}$ 的材料特性。图 4(d) 是该 电池循环性能图, 前期比容量稍有波动, 这可能由 于 CLASP 膜中的成分在循环过程中逐渐渗入正极 材料引起的。而随着充放电次数的增加, 比容量基 本稳定在 $117 \mathrm{mAh} / \mathrm{g}$ 左右, 电池的效率也基本保持 在 $96 \%$ 以上, 说明应用 CLASP 的全固态电池具有 良好的循环稳定性。

\section{3 结论}

通过与负载 $\mathrm{Al}_{2} \mathrm{O}_{3}$ 的无纺布膜复合, 制备了含 LAGP、PEO、SN 和 LiTFSI 的新型复合固态电解质 膜 CLASP, 并用它组装了全固态电池。电解质膜的 高温稳定性极佳, 在 $170^{\circ} \mathrm{C}$ 下加热 $2 \mathrm{~h}$ 几乎不发生变 形, 该电解质膜的室温电导率为 $3.66 \times 10^{-5} \mathrm{~S} / \mathrm{cm}$, 在 $100^{\circ} \mathrm{C}$ 时的电导率为 $2.52 \times 10^{-4} \mathrm{~S} / \mathrm{cm}$, 且电化学窗口 达 $5.5 \mathrm{~V}$, 循环稳定且效率高, 适合扩大生产, 且有 应用于高压正极材料的潜力。

\section{参考文献:}

[1] XUE Z G, HE D, XIE X L. Poly(ethylene oxide)-based electrolytes for lithium ion batteries. Journal of Materials Chemistry A, 2015, 3(7): 19218-19253.

[2] GOODENOUGH J B, KIM Y. Challenges for rechargeable Li batteries. Chemistry Materials, 2010, 22(3): 587-603.
[3] ZHENG Y L, CEHN R J, WU F, et al. Progress of research on the conductive mechanism of the glassy electrolytes in lithium ion batteries. Journal of Inorganic Materials, 2013, 28(11): 1172-1180.

[4] ZHOU W D, WANG S F, LI Y T, et al. Plating a dendrite-free lithium anode with a polymer/ceramic/polymer sandwich electrolyte. Journal of the American Chemical Society, 2016, 138(7): 9385-9388.

[5] CHEN B, HUANG Z, CHEN X T, et al. A new composite solid electrolyte $\mathrm{PEO} / \mathrm{Li}_{10} \mathrm{GeP}_{2} \mathrm{~S}_{12} / \mathrm{SN}$ for all-solid-state lithium battery. Electrochim. Acta, 2016, 210(6): 905-914.

[6] HU P, CHAI J C, DUAN Y L, et al. Progress in nitrile-based polymer electrolytes for high performance lithium batteries. Journal of Materials Chemistry A, 2016, 4(5): 10070-10083.

[7] LU Y, KORF K, KAMBE Y, et al. Ionic liquid nanoparticle hybrid electrolytes: applications in lithium metal batteries. Angewandte Chemie, 2014, 126(2): 498-502.

[8] CHEN X, USREY M, PENA H A, et al. Thermal and electrochemical stability of organosilicon electrolytes for lithium-ion batteries. Journal of Power Sources, 2013, 241(1): 311-319.

[9] ZHANG H, LIU C Y, ZHENG Li P, et al. Lithium bis (fluorosulfonyl)imide/poly(ethylene oxide) polymer electrolyte. Electrochimca Acta, 2014, 133(4): 529-538.

[10] ZHAO Y R, HUANG Z, CHEN S J, et al. A promising PEO/LAGP hybrid electrolyte prepared by a simple method for all-solid-state lithium batteries. Solid State Ionics, 2016, 295(8): 65-71.

[11] PITAWALA H M J C, DISSANAYAKE $\mathrm{M}$ A $\mathrm{K} \quad \mathrm{L}$, SENEVIRATNE V A. Combined effect of $\mathrm{Al}_{2} \mathrm{O}_{3}$ nano-fillers and EC plasticizer on ionic conductivity enhancement in the solid polymer electrolyte (PEO) ${ }_{9} L i T f$. Solid State Ionics, 2007, 178(4): 885-888.

[12] TARASCON J M, ARMAND M. Issues and challenges facing rechargeable lithium batteries. Nature, 2001, 414(11): 359-367.

[13] CHOI J H, LEE C H, YU J H, et al. Enhancement of ionic conductivity of composite membranes for all-solid-state lithium rechargeable batteries incorporating tetragonal $\mathrm{Li}_{7} \mathrm{La}_{3} \mathrm{Zr}_{2} \mathrm{O}_{12}$ into a polyethylene oxide matrix. Journal of Power Sources, 2015, 274(10): 458-463.

[14] SHARMA M V N V D, SARMA A V, RAO R B. Electrical conductivity, relaxation, and scaling analysis studies of lithium alumino phosphate glasses and glass ceramics. Journal of Materials Science, 2009, 44(2): 5557-5562.

[15] HUANG L Z, WEN Z Y, JIN J, et al. Preparation and characterization of PEO-LATP/LAGP ceramic composite electrolyte membrane for lithium batteries. Journal of Inorganic Materials, 2012, 27(3): 249-252.

[16] JIN L Y, JIANG Y X, LIAO H G, et al. FTIR spectroscopic studies of PEO-based polymer electrolyte with ionic liquid. Chemical Journal of Chinese University, 2009, 30(4): 767-771.

[17] YAN B G, ZHU Y Q, PAN F, et al. $\mathrm{Li}_{1.5} \mathrm{Al}_{0.5} \mathrm{Ge}_{1.5}\left(\mathrm{PO}_{4}\right)_{3}$ Li-ion conductor prepared by melt-quench and low temperature pressing. Solid State Ionics, 2015, 278(6): 65-68.

[18] MOHD R J, OON H S, SURIANI I, et al. Effects of $\mathrm{Al}_{2} \mathrm{O}_{3}$ nanofiller and EC plasticizer on the ionic conductivity enhancement of solid PEO- $\mathrm{LiCF}_{3} \mathrm{SO}_{3}$ solid polymer electrolyte. Solid State Ionics, 2011, 196(7): 41-47. 